\title{
Ecological and Phytochemical Studies on Brown Algae Sargassum muticum from Marsa Alam at Red Sea Coast, Egypt
}

\author{
Mohamed F. Azzazy ${ }^{1}$, Ayyad S.M², Ashraf M. Nofal ${ }^{3}$, Inas Z. Abdelsalam ${ }^{4}$, M. S. Abousekken ${ }^{5}$ and \\ Omar A.S. Tammam ${ }^{6}$
}

\begin{abstract}
The present investigation revealed that Red sea stretches along Marsa Alam of Egypt is a habitation of diverse groups of marine macro-algal species recorded along Marsa Hemera shore. Ecological studies (meteorological data, water, soil, aqueous extract analysis of Sargassum muticum (Yendo) Fensholt and associated species) reported that, slightly alkaline; low turbidity, moderate temperature and available nutrient content of saline water produced massive growth of $S$. muticum during September (2018). Ecological and Physico-chemical properties of collected water samples showed variations of different parameters of sea water like temperature, salinity, pH, and high dissolved minerals. Heavy metals accumulation inside the investigated seaweeds $S$. muticum was within the corresponding range. The aqueous extract of $S$. muticum contained high amounts of $\mathrm{Na}, \mathrm{Ca}, \mathrm{K}$ and $\mathrm{Fe}$, moderate amounts of $\mathrm{Zn}$, and $\mathrm{low}$ in $\mathrm{Cu}, \mathrm{Cd}$, $\mathrm{Ni}$ and Mn. Lead $\mathrm{Pb}$ recorded $0.291 \mathrm{ppm}, \mathrm{Ag}$, $\mathrm{Co}$ and Ga were absent in aqueous extract. The associated species to the brown algae $S$. muticum are belonging to 6 families and 7 species as follows: Sargassaceae, Fucaceae, Phaeophyceae, Rhodomelaceae, Caulerpaceae and Hydrocharitaceae. Results showed that $S$. muticum enriched in essential amino acids; micro and macro elements, carbohydrates, protein, lipids, and agar. These algae may be used as bio fertilizers.
\end{abstract}

Keywords: Seaweeds Ecology; Macro-algae, Red Sea coast, Marsa Alam, Phaeophyta, brown algae.

\section{INTRODUCTION}

Red Sea Governorate has an area of 130,000 km2. It includes some plains and highlands (Fig. 1). The weather in the plains is mainly dry and hot. The highlands are not populated. It is colder. In this district, the rainfall is very low and is not considered as water resource. The population growth rate of $2.09 \%$ is low compared to Egypt's mean which is $2.24 \%$ (Rayan et al

DOI: 10.21608/ASEJAIQJSAE.2019.6947

${ }^{1}$ Plant Ecologist, Surveys of Natural Resources Department,

Environmental Studies and Research institute, University of Sadat city

2. Phycologist, Botany Department, Mansoura University.

3. Microbiologist, Sustainable Development Deprtment,

Environmental Studies and Research institute, University of Sadat city

4. Medicinal and Aromatic Plants, Sustainable Development Deprtment,

Environmental Studies and Research institute, University of Sadat city.

5. Poultry nutrition, Sustainable Development Deprtment,

Environmental Studies and Research institute, University of Sadat city.

6. Wild Animal life, Surveys of Natural Resources Department,

Environmental Studies and Research institute, University of Sadat city.

Received August 06, 2019, Accepted December 30, 2019
2003). The marine environment in which seaweeds, brown algae exists possesses great taxonomic diversity and synthesis metabolites with interesting biological activities for food materials and in medical applications. The marine macro-algae grow in harsh environments, with variable water currents, a restricted nutrient supply, and high concentrations of salts, sunlight, and oxygen, which may foster the production of natural compounds (Poore, et al., 2013). The growth and chemical composition of marine macro algae are significantly affected by their environmental and habitat conditions. Physico-chemical factors determine the occurrence of particular seaweeds at particular place at a particular season as reported by (Jung, et al., 2013). Hence the study of the physico-chemical characters of marine ecosystem is also very important. Marine organisms, especially algae are rich sources of natural bioactive products. Seaweeds also have come up step by step starting with using them as food, later as raw material for industrial, medicinal, pharmaceutical and cosmetic purposes (Chennubhotla , et al., 2013), associated with their high contents of protein, polysaccharides, minerals, essential fatty acids, carotenoids and vitamins which are related to several environmental factors (Jeeva , et al., 2012; Polat \& Ozogul, 2013). The marine algae contain more than 60 trace elements, which are much higher than that in terrestrial plants and so, they have a pharmacological activities (Dhargalkar, 2014).

Seaweeds constitute an important source of natural resources for fertilizers and play an important role in agriculture and horticulture (Fan et al., 2011, Bierman and Rosen, 2013; Cordell and White, 2013, FAO, 2013). Seaweeds are known as sources of plant growth regulators, amino acids, mineral nutrients and vitamin precursors (Spinelli et al., 2010). The nutritional quality of seaweeds protein can be evaluated from amino acid composition and essential amino acids (Wong and 
Cheung, 2001). Their protein contents differ according to the species and seasonal conditions (Jadeja and Tewari, 2008). Agar, carrageenan and alginate are polysaccharides derived from seaweeds (Yang et al., 2011). Sargassum is a genus of seaweeds brown algae (order Fucales) macro-algae belonging to family Phaeophyceae, where they generally grow in shallow water and coral reefs. Most species within the Phaeophyceae are predominantly cold water organisms, but the genus Sargassum appears to be an exception (Hogan and Michael 2011).

Therefore, the present study was undertaken to investigate ecological parameters and phytochemical composition of brown algae seaweed, Sargassum muticum collected from Marsa Allam area (Marsa Hamera shores), Red Sea coast of Egypt during September, 2018.

\section{MATERIALS AND METHODS}

The resort of Marsa Alam is located on a town in south-eastern Egypt Red Sea (Fig. 1). Located 274 km south of Hurghada and connected to Edfu by $289 \mathrm{~km}$ long desert road. After creation of Marsa Alam International Airport in 2003, it became highly popular city. The Latitude: $25^{\circ} 4^{\prime} 3.4536 " \mathrm{~N}$, Longitude: $34^{\circ} 52^{\prime}$ 44.292" E

\section{Meteorological data}

Marsa Alam has a hot tropical desert climate like Hurghada and Sharm El Sheik. The collection site was along the semi-exposed shores of Marsa Alam and Marsa Hemera Red Sea coast of Egypt (Figs.1 and 2). It is one of the most important places of interest for brown algae growth in Egypt.

\section{Meteorological Studies}

Meteorological data obtained from nearest weather Station: El Qoseir, Egypt.

\section{Sampling}

Collection of brown algae (Sargassum sp.) and all associates.

\section{Taxonomical studies of the all sea weeds.}

The Identification of Sargassum sp. (Fig.3) and associated sea weeds. According to (Bhavanath et al 2009)

Separation of the different species according (De Széchy, 2012).

\section{Identification of the collected sea weeds.}

Sargassum sp. and its associated seaweeds were collected during September 2018 (Fig. 4 and 5). Macroalgae were collected using three quadrates $(10 \mathrm{~m} \times 10$ $\mathrm{m})$ in order to record each species. The identification and nomenclature of macro-algae followed (De Clerck,
2013, Bhavath et al 2009) and verified with illustrations by (De Széchy, 2012)

S. muticum, is commonly known as Japanese wire weed, large brown seaweed of the genus Sargassum. It is invasive seaweed with high growth rate. It has an efficient dispersion.

\section{Higher classification: Sargassum}

Order: Fucals

Class: Phaeophyceae

Family: Sargassaceae

Scientific name: Sargassum muticum (Yendo) Fensholt

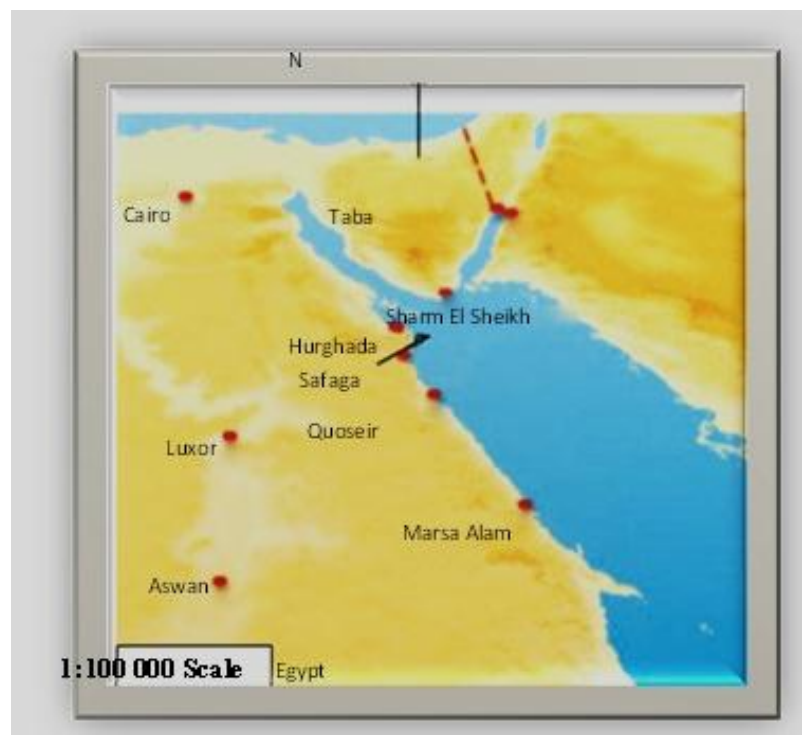

Fig.1. Map showing Red Sea coast of Egypt



Fig. 2. Show Red Sea coast and the study site of Marsa Hemera After Rayan et al 2003 


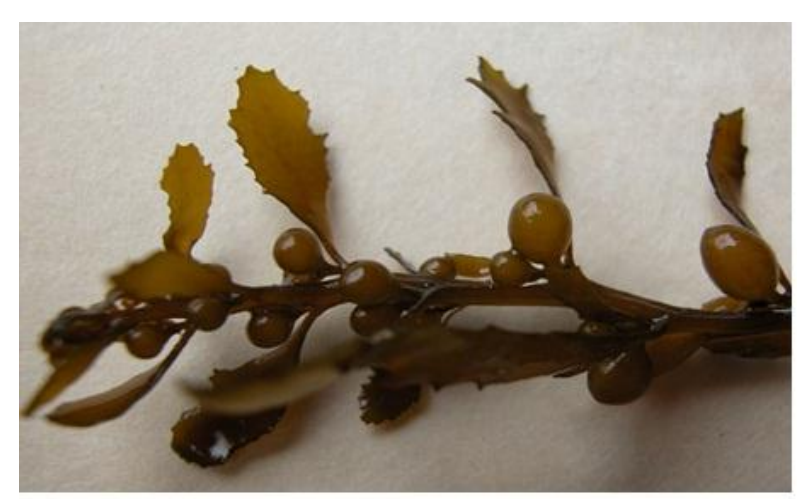

Fig. 3. Genral view of Sargassum muticum

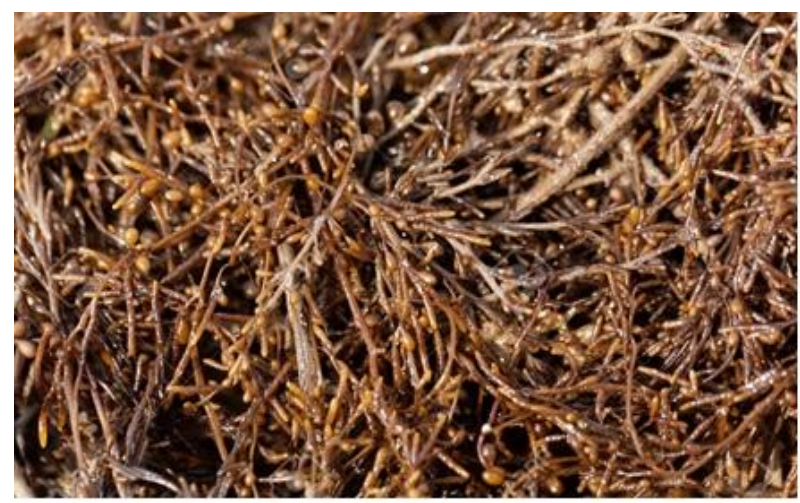

Fig. 4. Japaneese Wire weed

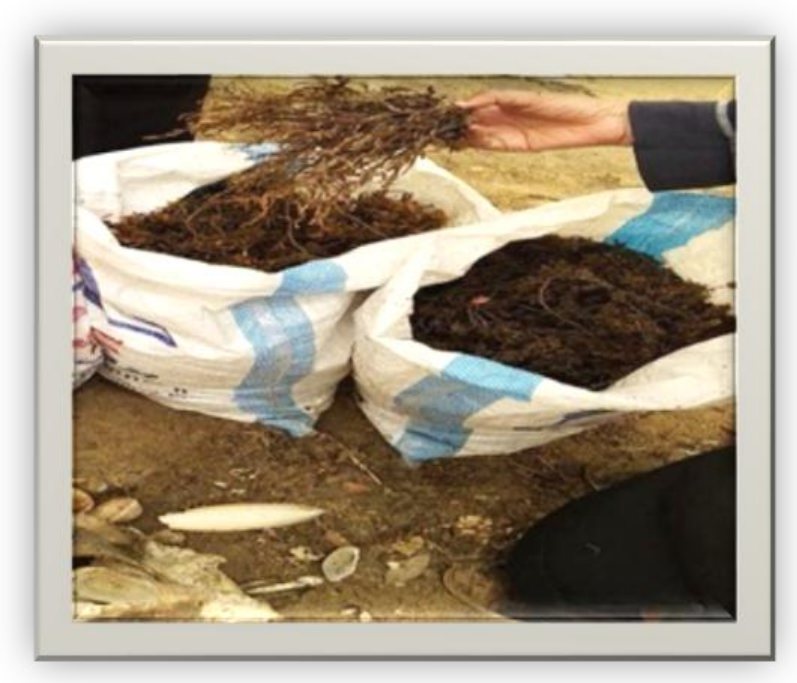

Fig. 5. Sargassum muticum (Japaneese Wire weed) collected from the Red Sea coast at Marsa Hemera

S. muticum is submerged macro-algae attached to the sea floor either on mud or rocks by hold fast has been collected. Seven other associated species of macroalgae have been found, collected and identified. They are belonging to 6 families.

\section{Collection and analysis of soil and water \\ Collection of soil and water samples}

Water samples were collected at a depth $50 \mathrm{~cm}$ below the surface, then filtered using membrane filter of $47 \mathrm{~mm}$ diameter and $0.45 \mu \mathrm{m}$ pore size, and were kept frozen at $-20^{\circ} \mathrm{C}$ for later analysis.

\section{Physico-chemical analysis of water}

Physicochemical parameters were measured according to (APHA, 1992) which including temperature, $\mathrm{pH}$, salinity, $\mathrm{K}, \mathrm{Na}, \mathrm{Ca}, \mathrm{Mg}$ and heavy metals were determined in lab of Soil Fertility Tests and Fertilizers Quality Control Laboratory Accredited according to ISO 17025/2005 Faculty of Agriculture, Mansoura University. The total contents of different elements were determined according to (Allen et al. 1997)

\section{Physico-chemical analysis of soil samples}

Soil samples were air dried and passed through a 2 $\mathrm{mm}$ sieve for eliminates gravels and coarse fragments. Analysis of soil samples included: texture, colour and calcium carbonate content. Texture and Calcium carbonate content analysis performed using standard hydrometer method according to (Carter and Gregorich, 2008). The soil colour was measured using Munsell soil colour charts (Munsell Colour Company, 1975).

\section{Preparation of $S$. muticum samples.}

S. muticum was washed with tap water and distilled water to remove salts on exposed surface, shade dried, and then kept in an oven at $60{ }^{\circ} \mathrm{C}$ for $4 \mathrm{~h}$, then ground and stored in polyethylene bags at room temperature.

\section{Chemical analysis of $\boldsymbol{S}$. muticum aqueous extract.}

Minerals $(\mathrm{Na}, \mathrm{Ca}, \mathrm{K}$ and $\mathrm{Mg})$ and $(\mathrm{Cu}, \mathrm{Co}, \mathrm{Zn}, \mathrm{Fe}$, $\mathrm{Cr}, \mathrm{Cd}$ and $\mathrm{Mn}$ ) heavy metals were determined using Lab of Soil Fertility Tests and Fertilizers Quality Control Laboratory Accredited according to ISO $17025 / 2005$ faculty of agriculture Mansoura university, using Thermo scientific iCap 7000 (Fig.6).

Protein content of $S$. muticum was determined using spectrophotometer followed (Bradford 1976), while, total carbohydrates were determined using spectrophotometer anthrone method (Hedge and Hofreiter 1962). The lipid content determined using the method following (Van Wychen et al, 2015), but the agar content extracted using method of (Roberts et al. 2015). 


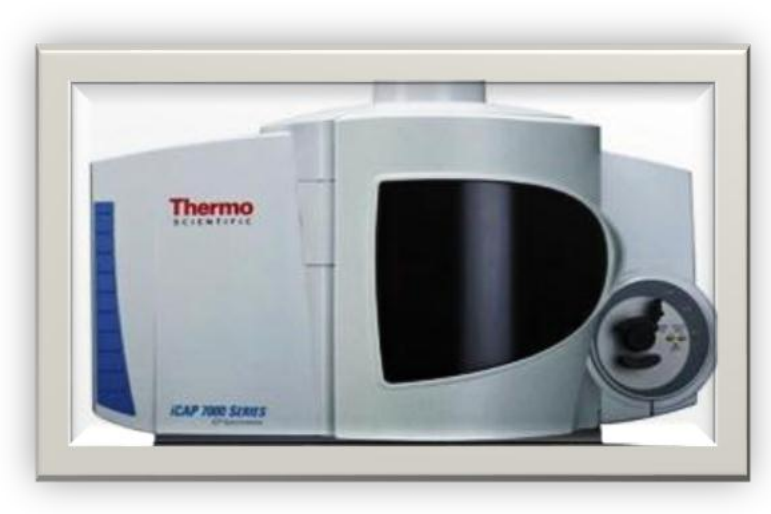

Fig.6 Thermo scientific iCap 7000

\section{Antioxidant activity of Sargassum species aqueous extract}

Detection method according to: Free radical scavenging of plant extracts was determined using ferric reducing antioxidant power assay (FRAP) procedure described by (Yu W et. al 2004).

\section{Statistical analysis}

All determinations were in triplicate. Data were expressed in terms of mean and were expressed in terms of mean \pm SD. While, analyzed for variance and the least significant difference (LSD) using One-way ANOVA $(P<0.05)$, (SPSS, 2007).

\section{RESULTS}

\section{Meteorological data of Marsa Alam area}

Climate and average monthly weather in Marsa Alam, Egypt
Data showed in Table 1 that; Air temperature (Max) varied from $29,31,32,33$ and $31^{\circ} \mathrm{C}$ in May, June, July, August, and September respectively. The months have a high chance of precipitation October, Novamber and December where $1 \mathrm{~mm}$ the rate of rainfall. The warmest (air temp) month is July and August 32 and $33^{\circ} \mathrm{C}$. October is the wettest month where the relative humidity $56 \%$.

\section{Taxonomical Studies of the collected species}

Identification of Sargassum sp. and associated seaweeds.

The morphological study of $S$. muticum showed that plants were dark brown, $30-200 \mathrm{~cm}$ in length, leaves were $6-8 \mathrm{~cm}$ length, shape: linear to ovate, apex: acute, midrib inconspicuous and having serrate margin.

The relative abundance of each individual was estimated according to the equation folowed (Ashraf, Salman and Mohamed Azzazy, 2013):

\section{Abundance \% \\ $=\frac{\text { No. individuals of a given species X } 100}{\text { Total No of all }}$}

Data in Table 2 revealed that, about 7 species belonging to 6 algal families identified at the Herbarium of the Environmental Studies and Research Institute, University of Sadat City during September 2018. The seaweeds community was quite diverse and dominated with $S$. cinereum Sargassaceae, with relative abundance $27.08 \%$ followed by $C$. myrica and $H$. johnsonii $14.58 \%$ for each, T. ornata $12.5 \%$ and L. papillosa recorded $10.41 \%$, and $P$. minor with relative aboundance $8.33 \%$, while C. racemosa recorded lowest representation $4.16 \%$.

Table 1. Average climatic factors in Marsa alam (Mersa Hemera) 2018/2019

\begin{tabular}{|c|c|c|c|c|c|c|}
\hline \multirow[t]{2}{*}{ Climate Item } & \multicolumn{2}{|c|}{$\begin{array}{c}\text { Temprature } \\
0^{\circ} \mathrm{C}\end{array}$} & \multirow{2}{*}{$\begin{array}{c}\text { Water } \\
\text { Temprature } \\
0^{\circ} \mathrm{C}\end{array}$} & \multirow[t]{2}{*}{$\begin{array}{c}\text { Rain fall } \\
\text { mm }\end{array}$} & \multirow[t]{2}{*}{$\begin{array}{l}\text { Wind speed } \\
\mathrm{Km} / \mathrm{h}\end{array}$} & \multirow{2}{*}{$\begin{array}{c}\text { Relative } \\
\text { humidity } \\
\%\end{array}$} \\
\hline & Max & Min & & & & \\
\hline January & 28 & 25 & 26 & 0 & 14.4 & $48 \%$ \\
\hline February & 22 & 14 & 22 & 0 & 14.4 & $48 \%$ \\
\hline March & 24 & 16 & 24 & 0 & 14.4 & $50 \%$ \\
\hline April & 27 & 19 & 23 & 0 & 14.4 & $50 \%$ \\
\hline May & 29 & 22 & 25 & 0 & 10.8 & $48 \%$ \\
\hline June & 31 & 25 & 26 & 0 & 14.4 & $48 \%$ \\
\hline July & 32 & 26 & 28 & 0 & 10.8 & $50 \%$ \\
\hline August & 33 & 26 & 29 & 0 & 10.8 & $51 \%$ \\
\hline September & 31 & 25 & 28 & 0 & 14.4 & $54 \%$ \\
\hline October & 29 & 22 & 27 & $1 \mathrm{~mm}$ & 7.2 & $56 \%$ \\
\hline November & 26 & 18 & 26 & $1 \mathrm{~mm}$ & 14.4 & $54 \%$ \\
\hline December & 23 & 15 & 24 & $1 \mathrm{~mm}$ & 14.4 & $54 \%$ \\
\hline
\end{tabular}

* Data obtained from nearest weather station: El Qoseir, Egypt (70.0 KM) 
Table 2: Analysis of the associated sea weeds to the brown macro algae Sargassum muticum (Yendo) Fensholt quantitatively

\begin{tabular}{|c|c|c|c|c|}
\hline Dominant Sp. & Associates & Family & $\begin{array}{l}\text { Number of } \\
\text { Individuals }\end{array}$ & $\begin{array}{c}\text { Abundance } \\
\%\end{array}$ \\
\hline \multirow{7}{*}{$\begin{array}{l}\text { Sargassum } \\
\text { muticum (Yen } \\
\text { do) Fensholt }\end{array}$} & Sargassum cinereum J.Agardh & Sargassaceae & 13 & 27.08 \\
\hline & $\begin{array}{l}\text { Turbinaria ornata (Turner) J. } \\
\text { Agardh }\end{array}$ & & 6 & 12.5 \\
\hline & $\begin{array}{l}\text { Cystoseira myrica (S.G. } \\
\text { Gmelin) C. Agardh }\end{array}$ & Fucaceae & 7 & 14.58 \\
\hline & Padina minor yamada & Phaeophyceae & 4 & 8.33 \\
\hline & $\begin{array}{l}\text { Laurencia papillosa } \\
\text { (C. Agardh) Greville }\end{array}$ & Rhodomelaceae & 5 & 10.41 \\
\hline & Caulerpa racemosa (C. Ag ardh) & $\begin{array}{l}\text { Caulerpaceae } \\
\text { Chlorophyta }\end{array}$ & 2 & 4.16 \\
\hline & $\begin{array}{l}\text { Halophila johnsonii Eiseman } \\
\text { (seagrass }\end{array}$ & Hydrocharitaceae & 7 & 14.58 \\
\hline
\end{tabular}

Table 3. Minerals and heavy metals of sea water sample



Table 4. Showed mean values of $\mathrm{pH}$, salinity, temperature and metal concentration $(\mathrm{lg} / \mathrm{L})$ in sea water samples at Marsa Alam (Marsa Hamera) Red Sea coast

\begin{tabular}{|c|c|c|c|c|c|c|}
\hline Parameter & Max & Min & Average & Median & $\mathbf{S D}^{\mathbf{a}}$ & $\mathrm{CV} \%^{\mathrm{b}}$ \\
\hline $\mathrm{pH}$ & 8.72 & 8.41 & 8.56 & 8.56 & 0.23 & 2.61 \\
\hline Salinity & 41.34 & 38.21 & 39.77 & 39.00 & 1.07 & 2.71 \\
\hline Temperature & 31.00 & 25.00 & 27.50 & 27.50 & 1.02 & 2.21 \\
\hline $\mathrm{Fe}$ & 8.99 & 5.75 & 5.93 & 6.02 & 1.41 & 14.63 \\
\hline $\mathrm{Mn}$ & 10.20 & 2.90 & 5.28 & 4.35 & 2.81 & 53.28 \\
\hline $\mathrm{Ni}$ & 10.10 & 6.70 & 8.65 & 9.05 & 1.16 & 13.44 \\
\hline $\mathrm{Cu}$ & 0.165 & 0.100 & 3.33 & 3.30 & 0.99 & 29.85 \\
\hline $\mathrm{Zn}$ & 5.70 & 0.94 & 2.62 & 2.40 & 1.70 & 64.98 \\
\hline $\mathrm{Cd}$ & 0.14 & 0.09 & 0.12 & 0.12 & 0.02 & 12.60 \\
\hline$\underline{\mathrm{Pb}}$ & 1.80 & 0.93 & 1.49 & 1.65 & 0.34 & 22.85 \\
\hline
\end{tabular}




\section{Physical and chemical analysis of Sargassum habitat Water}

Table 3 Showed that water sample recorded moderate temperature $\left(28^{\circ} \mathrm{C}\right)$, alkaline $(8.4)$ the relative high concentration of $\mathrm{Na}(1.75 \%)$ and $\mathrm{Cl}(1.59 \%)$. Higher mineral content was found of $\mathrm{Ca}, \mathrm{K}$ and $\mathrm{Al}$, $449.540,484.492$ and $90.848 \mathrm{ppm}$ respectively, the mineral contents of $\mathrm{Zn}, \mathrm{Cu}, \mathrm{Co}$, Fe were 0.010, 0.192, 0.013 and $0.038 \mathrm{ppm}$ respectively. The heavy metals contents in tested water sample of tested study were $(0.00 \mathrm{ppm})$ in case $\mathrm{Ag}, \mathrm{Ni}$ or very low amounts in case of Cd (0.02ppm).

Table 4 showed amounts of elements in water sample associated with the studied macro-algae. Data revealed that the highest value Potassium $(\mathrm{K})$ recorded $484.492 \mathrm{ppm}$, followed by by Ca (Calcium) 449.540 ppm, $\mathrm{Mg}$ (Magnesium) recorded. 1,102ppm. Iron (Fe) recorded $0.038 \mathrm{ppm}$, while Silver $(\mathrm{Ag})$, Boron $(\mathrm{B})$ and Bithmus (Bi) were not recorded in water sample.

Soil

Table 5 showed soil samples associated to $S$. muticum of study area have soil moisture $12.32 \%$ and the salinity was $3.00 \mathrm{dsm}^{-1}$, The chemical analysis of soil samples in Table 5 showed that have moderate alkaline $(\mathrm{pH}=8.47)$. The real density and Virtual density were 1.80 and $1.14 \mathrm{~g} / \mathrm{cm} 3$ respectively.

Data obtained in Table 6, Showed the soluble cations contents $\mathrm{K}^{+}, \mathrm{Na}^{+}, \mathrm{Ca}^{+2}$ and $\mathrm{Mg}^{+2}$ were 0.68 , $18.64,4.16$ and $19.39 \mathrm{meq} / 100 \mathrm{~g}$ respectively. Also, the soluble anions of $\mathrm{Cl}^{-}, \mathrm{So}_{4}^{-2}, \mathrm{H} \mathrm{Co}_{3}{ }^{-}$and $\mathrm{Co}_{3}{ }^{-2}$ were $19.92,10.17,1.71$ and $0.0 \mathrm{meq} / 100 \mathrm{~g}$ respectively.

\section{Biochemical analysis of $S$. muticum}

Table 7, showed the biochemical analysis of the dominant macro-algae S. muticum during September 2018, data revealed that; total carbohydrates $35.37 \pm$ $0.40 \mathrm{mg} / \mathrm{g}$ dry weight, protein content recorded $3.20 \pm$ 0.30 ; lipids recorded $2.80 \pm 0.04 \mathrm{mg} / \mathrm{g}$ while, the agar content recorded $91.37 \pm 1.20 \mathrm{mg} / \mathrm{g}$ of dry weight.

Table 8, showed the amounts of elements in aqueous extract of $S$. muticum Data revealed Table 8 that the highest value $21258.86 \mathrm{ppm}$ recorded by $\mathrm{Na}$ (Sodium) followed by Mg (Magnesium) $878.064 \mathrm{ppm}$; Potassium (K) recorded 5,469.139 ppm; Phosphorus (P) recorded $379.0905 \mathrm{ppm}$, Iron $(\mathrm{Fe})$ recorded 8.990 ppm while Silver (Ag), Cobalt (Co) and Gallium (Ga) were not recorded.

Table5. Physical analysis of soil

\begin{tabular}{cccccc}
\hline NO & Humidity (\%) & Real denisity $\mathbf{g} / \mathbf{~ c m}^{-\mathbf{3}}$ & Virtual density $\mathbf{~} / \mathbf{c m}^{-\mathbf{3}}$ & $\begin{array}{c}\text { Salinity } \\
\mathbf{d S m}^{-\mathbf{1}}\end{array}$ & $\mathbf{p H}$ \\
\hline 2 & 12.32 & 1.80 & 1.14 & 3.00 & 8.47 \\
\hline
\end{tabular}

Table 6. Chemical analysis of soil

\begin{tabular}{cccccccc}
\hline & \multicolumn{3}{c}{ Soluble Cations meq/100g } & \multicolumn{3}{c}{ Soluble Anions meq/100g } \\
\hline $\mathbf{K}^{+}$ & $\mathbf{N a}^{+}$ & $\mathbf{C a}^{+2}$ & $\mathbf{M g}^{+2}$ & $\mathbf{C l}^{-}$ & $\mathbf{S O}_{4}^{-2}$ & $\mathbf{H C O}_{3}^{-}$ & $\mathbf{C O}^{-{ }^{-2}}$ \\
\hline 0.68 & 18.64 & 4.16 & 19.39 & 19.92 & 10.17 & 1.71 & $\cdots$ \\
\hline
\end{tabular}

Table 7. Total carbohydrates, Protein, Lipids and agar of $S$. muticum mg/g dry weight

\begin{tabular}{clccc}
\hline Item & Carbohydrates & Protein & Lipids & Agar \\
\hline September 2018 & $35.370 \pm 0.40$ & $3.20 \pm 0.30$ & $2.80 \pm 0.04$ & $91.37 \pm 1.20$ \\
\hline
\end{tabular}

Table 8. Analysis of the minerals aqueous extract of S. muticum during September 2018

\begin{tabular}{|c|c|c|c|c|c|c|c|c|c|c|c|c|c|c|c|c|c|c|c|c|c|c|c|c|c|c|c|}
\hline 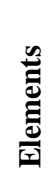 & 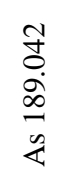 & 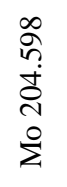 & 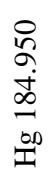 & $\begin{array}{l}\infty \\
0 \\
0 \\
\infty \\
0 \\
0 \\
\infty \\
\mathbb{Z}\end{array}$ & 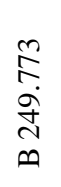 & 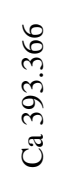 & 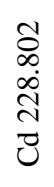 & 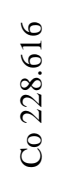 & 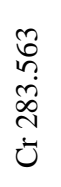 & 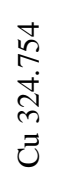 & 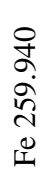 & 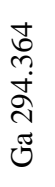 &  & 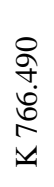 & 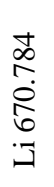 & $\begin{array}{l}n \\
\hat{n} \\
2 \\
\hat{\lambda} \\
n^{\infty}\end{array}$ & $\begin{array}{l}0 \\
b \\
i \\
i \\
i \\
\Sigma\end{array}$ & 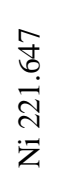 & $\begin{array}{l}\tilde{n} \\
\tilde{\Omega} \\
\hat{N} \\
\hat{2} \\
\hat{2}\end{array}$ & $\begin{array}{l}\stackrel{ }{\stackrel{1}{0}} \\
\hat{+} \\
\dot{\omega}\end{array}$ & 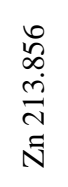 &  & 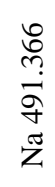 & 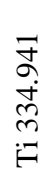 & $\begin{array}{l}\stackrel{\sim}{n} \\
\stackrel{n}{n} \\
\stackrel{2}{z}\end{array}$ & $\begin{array}{l}\vec{\Xi} \\
\ddot{j} \\
\vec{\lambda} \\
\vec{n}\end{array}$ & $\begin{array}{l}\text { ב. } \\
\text { ב. }\end{array}$ \\
\hline  & 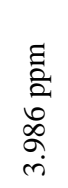 & 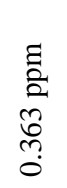 & 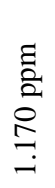 & $\stackrel{\rho}{\mathrm{Z}}$ & 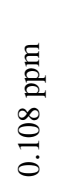 & $\begin{array}{l}\stackrel{M}{=} \\
\vec{i} \\
\stackrel{\infty}{=}\end{array}$ & 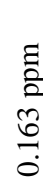 & คి & 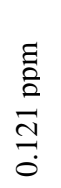 & 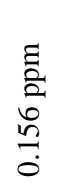 & $\begin{array}{l}\Xi \\
\vdots \\
\vdots \\
\stackrel{2}{2} \\
\infty \\
\infty\end{array}$ & $\stackrel{\rho}{\mathrm{Z}}$ & 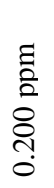 & $\begin{array}{l}\text { ले } \\
\stackrel{0}{8} \\
\dot{0} \\
\text { in }\end{array}$ & 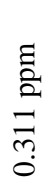 & 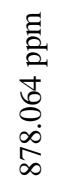 & $\begin{array}{l}\stackrel{\Xi}{2} \\
\stackrel{2}{2} \\
\stackrel{+}{0} \\
\stackrel{0}{0}\end{array}$ & 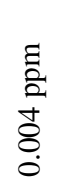 & 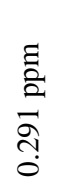 & 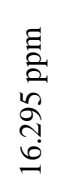 & 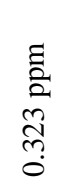 & 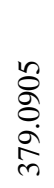 &  & 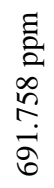 & 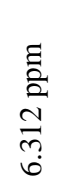 & 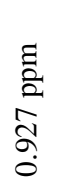 & $\begin{array}{l}\text { ?) } \\
\stackrel{0}{0}\end{array}$ \\
\hline
\end{tabular}


Table 9. Mean values of heavy metals (ppm) in brown algae S. muticum aqueous extract at Marsa Alam (Marsa Hemera) Red Sea

\begin{tabular}{ccccccc}
\hline Metal & Min & Max & Average & Median & SD $^{\mathbf{a}}$ & CV\% $^{\mathbf{b}}$ \\
\hline $\mathrm{Cr}$ & 0.121 & 0.142 & 0.1315 & 0.1315 & 0.48 & 1.03 \\
$\mathrm{Mn}$ & 0.754 & 2.912 & 1.833 & 1.833 & 72.51 & 47.20 \\
$\mathrm{Ni}$ & 0.004 & 1.31 & 0.657 & 0.657 & 1.22 & 18.01 \\
$\mathrm{Cu}$ & 0.192 & 1.75 & 0.971 & 0.971 & 2.97 & 46.25 \\
$\mathrm{Zn}$ & 0.64 & 0.323 & 0.481 & 0.481 & 2.34 & 71.38 \\
$\mathrm{Cd}$ & 0.33 & 0.163 & 0.246 & 0.246 & 0.05 & 21.25 \\
$\mathrm{~Pb}$ & 0.51 & 0.291 & 0.400 & 0.400 & 0.24 & 18.12 \\
\hline
\end{tabular}

While, a Standard deviation ${ }^{\mathbf{b}}$ Coefficient of variation

Data in Table 9 showed elements in aqueous extract of S. muticum where, highest value $2.912 \mathrm{ppm}$ recorded by Manganeze $(\mathrm{Mn})$, while the lowest value 0.163 ppm Cadmium(Cd).

Table 10 revealed that Glutamine amino acid recorded the highest value $0.38 \%$ followed by Asprteine 0.355 , while the lowest value recorded by cysteine $0.04 \%$ of the dominant macro-algae species $S$. muticum aqueous extract.

Table 10. Amino acids analysis of $S$. muticum aqueous extract

\begin{tabular}{cc}
\hline Parameters & \% crude protein \\
\hline Asprteine & 0.35 \\
Threonine & 0.16 \\
Serine & 0.15 \\
Glutamine & 0.38 \\
Glycine & 0.22 \\
Alanine & 0.28 \\
Valine & 0.19 \\
Isoleucine & 0.15 \\
Leucine & 0.22 \\
Tyrosine & 0.14 \\
Phenylalanine & 0.16 \\
Histedine & 0.05 \\
Lysine & 0.15 \\
Argenine & 0.16 \\
Proline & 0.11 \\
Cysteine & 0.04 \\
Methionine & 0.07 \\
\hline
\end{tabular}

Table 11 represent the antioxidant activity of $S$. muticum aqueous extract showed that, DPPH recorded highest value $54.74 \pm 0.59 \%$ and $54.32 \pm 0.47 \%$ at concentrations 200 and $150 \mathrm{ug} / \mathrm{ml}$ respectively, while the lowest value $51.88 \pm 0.27 \%$ at concentration $50 \mathrm{ug} / \mathrm{ml}$.
Table 11. Antioxidant of $S$. muticum aqueous extract

\begin{tabular}{ccc}
$\begin{array}{c}\text { Sample } \\
\text { I.D }\end{array}$ & $\begin{array}{c}\text { Sample } \\
\text { Concentration } \\
(\mathbf{u g} / \mathbf{m l})\end{array}$ & $\begin{array}{c}\text { DPPH \% } \\
\text { "RSA" }\end{array}$ \\
\hline & 50 & $51.88 \pm 0.27 \%$ \\
Aqueous & 100 & $53.56 \pm 0.39 \%$ \\
extract of & 150 & $54.32 \pm 0.47 \%$ \\
S. muticum & 200 & $54.74 \pm 0.59 \%$ \\
\hline
\end{tabular}

\section{DISCUSSION}

The environmental factors can become a stress to a given seaweed species, if it exceeds the upper or lower threshold values of tolerance (Hurd et al 2014). Marine organisms grow in often distinctive vertical or horizontal "zones" or "bands" along these gradients, thereby providing "natural laboratories" in which to study environmental (abiotic) and biological processes shaping the communities (Raffaelli and Hawkins 1996). $S$. muticum grows from half-tide to infra littoral areas (to a depth of $10 \mathrm{~m}$ (Thomas2002). Water transparency have profound effects on the quantity and quality of the light reaches seaweeds at their growth sites, where the primary importance of light to seaweeds is in providing the energy for photosynthesis (Huard et al 2014).

The temperature of the studied site during September 2018 varied from $33.00 \pm 0.21$ to 25.00 $\pm 0.21^{\circ} \mathrm{C}$ (Table 1). Temperature affects the growth stages of macro- algae as tide level. Temperature is considered as a vital environmental factor controlling the growth and metabolic rates of marine organisms, especially on metabolic processes of photosynthesis and respiration in macro- algae (Zou and Gao, 2014). The physicochemical analysis of water indicates such as relative decrease of temperature, high alkalinity and salinity from September to November were parallel with Egyptian climate where water evaporation decreased 
and ran off beginning during October and increased during November (Jeeva et al, 2012).

The present study revealed that, macro- algal community was quite diverse with 6 families and 7 species recorded at Red Sea coast at Marsa Alam (Mersa Hemera study area), Sargassaceae represented by (S. muticum, S. cinereum and $T$. ornate). Fucaceae (C. myrica), Phaeophyceae (P. minor), Rhodomelaceae (L. papillosa), Chlorophyta Caulerpaceae (C. racemose) and Hydrocharitaceae (H. johnsonii) (Negm, 1988). S. muticum recorded the dominant community were associates represented with $S$. cinereum abundance $27.08 \%$ of the total associated macro-algae during September Table 2, this findings agreed with (Nazni and Renuga, 2015) who reported that the highest growth of Sargassum sp. recorded in September and October on the semi-exposed shore in Thailand. $S$. muticum is brown seaweed uses energy from sunlight to perform photosynthesis due to aerial vesicles allows the algae to raise to the sea water surface (Mohamed Abdel-Kareem, 2009).

The relative content of carbohydrates $(35.370 \pm$ $0.40)$, protein $(3.20 \pm 0.30)$, lipids $(2.80 \pm 0.04)$ and agar $(91.37 \pm 1.20) \mathrm{mg} / \mathrm{g}$ dry weight were recorded in Sargassum muticum extract during mature stage of alga in september Table 7. The relative low content of protein, lipids in $S$. muticum aqueous extract may due to relative low trophic (oligo-mesotrophic) status of the Red Sea water. Natural products such as agar content $(91.37 \pm 1.20) \mathrm{mg} / \mathrm{g}$ dry weight in $S$. muticum was agreed with that obtained by (Chee et al.2011) in Sargassum sp. in Malaysia. Minerals analysis of $S$. muticum showed higher contents of $\mathrm{Na}, \mathrm{K}$ and $\mathrm{Ca}$ during September Table 8, this high value of $\mathrm{Ca}$ may due to the geographic changes of sediment and shores (Zubia et al. 2014).

Table 9 showed that heavy metals contents in $S$. muticum aqueous extract were higher during September. This may due to the long-term variations of heavy metals level in the marine environment. Metal content in macro-algae depends on various biological and environmental factors (the concentration and availability of elements in water, interactions between chemical elements, temperature, salinity, $\mathrm{pH}$, light intensity and area geology) (Wasi and Ahmed 2013). Lead content $(\mathrm{Pb})$ in surrounding water were relatively high. (Al-Homaidan 2006) reported that, $\mathrm{Pb}$ in brown algae from the Arabian Gulf were higher than those from the Sudanese Red Sea coast. High levels in the Arabian Gulf could be attributed to more man made activities, in particular oil industry, than in the Red Sea. The considerable high content of heavy metals and minerals in $S$. muticum Table 8 than that found in sea water Table 3 may due to algal accumulation of heavy metals. This may attribute to the presence of charged polysaccharides and alginic acid in cell walls of brown seaweeds. (Murugaiyan and Narasimman, 2012).

Table 10 represented amino acids of $S$. muticum aqueous extract, data revealed that, Glutamine amino acid recorded highest value $0.38 \%$ followed by Asprteine 0.355 , while the lowest value recorded by cysteine $0.04 \%$ of the dominant macro-algae species $S$. muticum aqueous extract. In this connection, (Mattioli et al,2009) reported that, the growth enhancement effect of proline amino acid is mainly due to its role in regulating gene expression and providing hydroxyproline-rich glycoproteins that serve as structural constituents of the cell wall. Considering seaweeds as a rich source of proteins according to (Angell et al., 2016) the levels of different amino acids were profiled in plants supplemented with Sar and Jan extracts, while aspartate serves as an intermediate to support the synthesis of other amino acids, including lysine, threonine, isoleucine and methionine (Viola, 2001). The antioxidant property of the aqueous sea weed Sargassum muticum extract is shown in Table 11. The aqueous extracts of $S$. muticum capable of scavenging hydrogen peroxide in an amount dependent manner. $200 \mathrm{ug} / \mathrm{ml}$ of aqueus extract exhibited $54.74 \pm$ $0.59 \%$ scavenging activity on hydrogen peroxide. The results of antioxidant activity of different assays (DPPH, $\mathrm{H}_{2} \mathrm{O}_{2}$ ) showed that, the brown sea weed $S$. muticum has a higher radical scavenging property. Our presented study revealed that, the brown seaweed $S$. muticum aqueous extract showed excellent antioxidant activity as reported earlier (Hedge and Hofreiter, 1962). The antioxidant activity of brown seaweed extracts may due to their polyphenol content which might be the cause of recorded inhibitory effects (Van Wychen et al, 2015).

\section{CONCLUSION}

Our study indicated that, the physicochemical studied Red Sea water at Marsa Alam (Marsa Hemera) recorded moderate water temperature, slight alkaline $\mathrm{pH}$; low turbidity, and valuable nutrient contents of the saline water which exerted massive growth of $S$. muticum (Yendo) Fensholt with maximum abundance during September 2018. Six macroalgae families were recorded and dominated with Sargassaceae family $(S$. muticum). The parameters evaluated in this study help in identification and standardization of seaweed $S$. muticum. Biochemical analysis of $S$. muticum aqueous extract showed its content of vital components (carbohydrates, protein, amino acids, minerals and metals). This seaweed may be valuable as bio fertilizer and in pharmaceutical and food industry. Also, biological evaluation using human and animal feeding investigations would be required to establish 
the expected nutritional and therapeutically values of this seaweed.

\section{ACKNOWLEDGMENT}

Authors like to express their deep thanks to all participants, for valuable advice, guidance throughout the whole work. The present work was supported by a grant from the Research support system of the University of Sadat City, Egypt as a part of the Research Program.

Funding: This study was done with authors funding from The Research project No.9 (2017) from University of Sadat City, Egypt.

Conflict of Interest: The authors declare that there is no conflict of interest with others.

\section{REFERENCES}

Allen, L.B., P.H.Sitonen and H.C. Thomposon. 1997. Methods for the determination of arsenic, cadmium, copper, lead and tin in sucrose, corn syrups and high fructose corn syrups by inductively coupled plasma atomic emission spectrometry. J. of Agricultural and Food Chemistry. 45: 162- 165.

Ali, A. Al- Homaidan. 2006. Brown algae as biomonitors of heavy metal pollution along the Saudi coast of the Arabian Gulf. Saudi J. of Biological Sci. 13(13):99-103.

Angell, A. R., L. Mata, R. de Nys and N. A. Paul. 2016. The protein content of seaweeds: a universal nitrogen-toprotein conversion factor of five. J Appl Phycol. 28:511524.

APHA, American Public Health Association. 1992. Standard methods for the examination of water and wastewater. Washington DC: American Public Health Association. p. 18.

Ashraf, A. S. and F. A. Mohamed. 2013. Pollen Grains Indicators to Plant Habitat Conditions at Some Arid Regions Sadat Area Egypt. CATRINA. 8 (1): 21-28.

Bhavanath, J., C.R. K. Reddy, M.C. Thakur and M. U. Rao. 2009. Seaweeds of India; The diversity and Distribution of Seaweeds of the Gujarat Coast: Doi 10.1007/978-90-4812488-6 Springer Dordrecht Heidelberg London, New York.

Bierman, M.P. and J.C.Rosen. 2013. Nutrient cycling and maintaining soil fertility in fruit and vegetable crop systems. University of Minnesota Extension

Bradford, M. M. 1976. A rapid and sensitive method for the quantification of microgram quantities of protein utilizing the principle of protein-dye binding; Anal Biochem. 72: 248-54.

Carter, M. R. and E.G. Gregorich. 2008. Soil Sampling and Methods of Analysis (New York: Taylor and Francis)

Chee, S.Y., P.K. Wong and C.L.Wong. 2011. Extraction and characterisation of alginate from brown seaweeds (Fucales, Phaeophyceae) collected from Port Dickson, Peninsular Malaysia. J Appl Phycol. 23: 191-6.
Chennubhotla, V.S.K. and M.U. Rao, K.S..Rao. 2013. Commercial importance of marine macro algae. Seaweed Res Utiln, 35(2): 118-28.

Cordell, D. and S. White. 2013. Sustainable Phosphorus Measures: Strategies and technologies for achieving phosphorus security. Agronomy, 3(1): 86-116.

De Clerck, O., M.D. Guiry, F. Leliaert, Y. Samyn and H.Verbruggen. 2013. Algal taxonomy: a road to nowhere? J Phycol. 49: 215-25.

De Széchy, M.T.M., P.M. Guedes, M.H. Baeta-Neves and E.N.Oliveira. 2012. Verification of Sargassum natans (Linnaeus) Gaillon (Heterokontophyta: Phaeophyceae) from the Sargasso Sea off the coast of Brazil, Western Atlantic Ocean. Check List. 8: 638-41.

Deyab, M.A. and F.M. Ward. 2016. Ecological and biochemical analyses of the brown algae Turbinaria ornata (Turner) J. Agardh from Red Sea coast, Egypt. Journal of Coastal Life Medicine. 4(3):187-192.

Dhargalkar, V.K. 2014. Uses of seaweeds in the Indian diet for sustenance and well-being. Sci Cult. 80: 192-202.

Fan, D., M. Hodges, J. Zhang, C.W. Kirby, S.J. Locke, A.T. Critchley and A. Prithiviraj. 2011. Commercial extract of the brown seaweed Ascophyllumnodsum enhances phenolic antioxidant content of spinach which protects Caenorhabditiselegans against oxidative and thermal stress. Food Chemistry. 124: 195-202.

FAO. 2013. Edible insects: Future prospects for food and feed security. FAO Forestry. Paper 171.

Hedge, J.E., B.T. Hofreiter. 1962. Carbohydrate chemistry. 17th ed. New York: Academic Press.

Hogan, C. M., E.Monosson and C.J. Cleveland (eds.). 2011."Algae. Brown_algae". Encyclopedia of Earth Washington DC: National Council for Science and the Environment.

Hurd, C., P. Harrison, K.Bischof and C. Lobban. 2014. Physico-chemical factors as environmental stressors in seaweed biology; In Seaweed Ecology and Physiology (pp. 294-348). Cambridge: Cambridge University Press. doi:10.1017/CBO9781139192637.008.

Jadeja, R.N. and A. Tewari. 2008. Effect of soda ash industry effluent on protein content of two seaweeds; Journal of Hazardous Materials. 151:559561.

Jeeva, S., J.M. Antonisamy, C. Domettila, B. Anantham and M.Mahesh. 2012. Preliminary phytochemical studies on some selected seaweeds from Gulf of Mannar, India. Aisan Pac J Trop Biomed. 2(Suppl 1): S30-3.

Jung, K.A., S.R. Lim, Y. Kim, J.M. Park. 2013. Potentials of macroalgae as feedstocks for biorefinery. Bioresour Technol. 135: 182-90.

Mattioli, R., P.Costantino and M. Trovato. 2009. Proline accumulation in plants: not only stress. Plant Signal Behav. 4:1016-1018.

Mohamed, S.M. A -K. 2009. Phenetic Studies and New Records of Sargassum Species (Fucales, Phaeophyceae) from the Arabian Gulf Coast of Saudi Arabia Academic J. of Plant Sci. 2 (3): 173-181. 
Munsell Colour Company. 1975. Munsell Soil Colour Charts (Baltimore, MD: Macbeth Division of Kollmorgen Corporation).

Murugaiyan, K. and S. Narasimman. 2012. Elemental composition of Sargassum longifolium and Turbinaria conoides from Pamban Coast, Tamilnadu. Int J Res Biol Sci. 2: 137-40.

Nazni, P., Renuga. 2015. Nutrient composition of the selected brown seaweeds from Mandapam Coastal Regions; Southeast Coast of India. Int J Agric Food Sci. 2: 38-42.

Negm, S.N.M. 1988. Ecological, biological and phytochemical studies on some marine algae from the Red Sea coast of Egypt [dissertation]. Cairo: Cairo University.

Polat, S. and Y.Ozogul. 2013. Seasonal proximate and fatty acid variations of some seaweeds from the northeastern Mediterranean coast. Oceanologia. 55: 375-91.

Poore, A.G., A. Graba-Landry, M. Favret, B. H.Sheppard, M. Byrne and S.Dworjanyn. 2013. Direct and indirect effects of ocean acidification and warming on a marine plantherbivore interaction. Oecologia. 173: 1113-24.

Rayan, M.A., B.Djebedjian, I.Khaled and E.S. Salah. 2003. Desalination Option within Water Demand Management and Supply for Red Sea Coast in Egypt" Seventh International Water Technology Conference, IWTC. 2003. Cairo, Egypt

Roberts, D.A., N.A. Paul, S.A. Dworjanyn, Y. Hu, M.I. Bird and R. de Nys. 2015. Gracilaria waste biomass (sampah rumput laut) as a bioresource for selenium biosorption. $J$ Appl Phycol. 27: 611-20.

Raffaelli, D. and S. Hawkins. 1996. "Intertidal Ecology," Chapman \& Hall, London, doi: 10.1007/978-94-009-1489

Spinelli, F., G. Fiori, M. Noferini, M. Sprocatti and G.Costa. 2010. A novel type of seaweed extract as a natural alternative to the use of iron chelates in strawberry production: Scientia Horticulturae.125:263-269.
SPSS Base 15.0 User's Guide 2007. Chicago, IL: SPSS Inc.

Thomas, D. N. 2002. Seaweeds. The Natural History Museum, London

Van Wychen, S., K. Ramirez and L.M.L.Laurens. 2015. Determination of total lipids as fatty acid methyl esters (FAME) by in situ transesterification. Golden: National Renewable Energy Laboratory. p. 5. [Online] Available from: http://www.nrel.gov/docs/fy16osti/60958.

Wasi, S., S. Tabrez and M.Ahmad. 2013. Toxicological effects of major environmental pollutants: an overview. Environ Monit Assess. 185: 2585-93.

Viola, R. E. 2001. The central enzymes of the aspartate family of amino acid biosynthesis. Acc Chem Res. 34:339-349

Wong, K.H. and P.K. Cheung. 2001. Nutritional evaluation of some subtropical red and green seaweed; Part I. Proximate composition, amino acid profile and some physicochemical properties; Food Chemistry. 71: 475-482.

Yang, B., G. Yu, X. Zhao, W. Ren, G. Jiai, L. Fang, Y. Wang, G. Du, C .Tiller, G. Gabrielle, C.J. Barrow, S. Ewart and J. Zhang. 2011. Structural characterization and bioactivities of hybrid carrageenan like sulphated galactan from red alga Furcellarialumbricalis. Food Chemistry. 124: 50-57.

Yu, W., Y. Zhao and B. Shu. 2004. The radical scavenging activities of radix puerariae isoflavonoids: A chemiluminescence study. Food Chem. 86: 525-529. doi: 10.1016/j.foodchem.08.037. [CrossRef] [Google Scholar]

Zou, D.H. and K.S. Gao. 2014. The photosynthetic and respiratory responses to temperature and nitrogen supply in the marine green macroalga Ulva conglobata (Chlorophyta). Phycologia. 53: 86-94.

Zubia, M., C.P. Payri, E. Deslandes and J. Guezennec. 2014. Chemical composition of attached and drift specimens of Sargassum mangarevense and Turbinaria ornata (Phaeophyta: Fucales) from Tahiti, French Polynesia. Bot Mar. 6: 562-71. 


\section{الملخص العربي}

\section{دراسات بيئية وفيتوكيميائية على طحلب السارجاسوم ميوتيكم من الطحالب البنية لساحل البحر الأحمر،}

\section{مصر}

$$
\text { محمد عزازى، سكينة عياد، اشرف نوفل، ايناس زكريا، محمود ابوسكين عمر تمام }
$$

الهيدروجيني، والمعادن الذائبة. اثبتت الدراسة أن الأعثاب

البحرية غنية بالأحماض الأمينية الأساسية والعناصر

الصغرى. وتتتمي مجموعة الطحالب المرافقة والتي تم جمعها

خلال هذه الدراسة إلى ست عائلات وهى: Sargassaceae,

Fucaceae, Phaeophyceae, Rhodomelaceae,

Caulerpaceae and Hydrocharitaceae

البحرية محل الدراسة V انواع كالتالى: Sargassum

muticum, S. cinereum, Turbinaria ornate, Cystoseira myrica, Padina minor, Laurencia papillosa, Caulerpa racemose and Halophila johnsonii.

أظهرت النتائج أن هذه الأنواع من الطحالب البحرية

تحتوي على نسبة جيدة من الكربوهيدرات ذات القيمة الغذائية

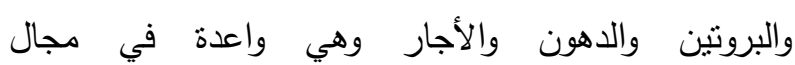

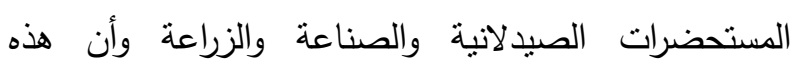

الطحالب لها أهمية اقتصادية ليس فقط كعلف ولكن أيضًا

$$
\text { كأسمدة حيوية. }
$$

كثفت هذه الدراسة على منطقة البحر الاحمر بطول

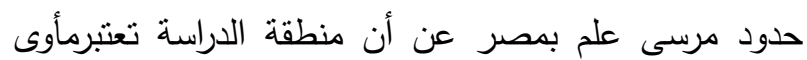
لمجموعات متنوعة من الأنواع البحرية الطحلبية المسجلة على طول شاطئ مرسى علم خصوصا منطقة مرسى حميرة حيث تتمو مجتمعات المانجروف التى بدورها تمد الأعثاب هرئى مرنى البحرية وجميع الكائنات المحيطة بالغذاء، وهذه المجموعة من بن الطحالب تنتمي إلى فايوفيتا ورودوفيتا وكلوروفيتا. واظهرت الدراسات البيئية ان المنطقة تتميز باعثدال فى درجات الحرارة ، ودرجة الحموضة والقلوية طفيفة. اثبتت الدراسة ايضا أن نقاء المياة وتوافر المغذيات للمياه المالحة ادت إلى نمو هائل Sargassum muticum وسيادة لنوع من الطحالب وهو (Yendo) Fensholt سبتمبر ^1 ـ Y. أظهرت الخصائص البيئية خصوصا الفيزيائية والكيميائية لمياه البحر الأحمر اختلافات في الخصائص للمياه السطحية مثل درجة الحرارة، الملوحة، الرقم 